\title{
Off-Pump Coronary Artery Bypass in a Patient with Severe Atherosclerosis in the Accessory Left Anterior Descending Artery
}

\author{
Lai Wei, MD, Jiasi Zhu, MD, Limin Xia, MD, Zhaohua Yang, MD, Huan Liu, MD, \\ and Chunsheng Wang, MD
}

\begin{abstract}
It is rare for an accessory left anterior descending artery to be the single coronary artery anomaly in adult with severe atherosclerosis. An off-pump coronary artery bypass was performed electively for the patient. We indentified that the accessory left anterior descending artery, accompanied by a little portion of the left anterior descending artery, ran in the anterior interventricular groove to the apex. Four vessel-anastomoses, including the left internal mammary artery to the accessory left anterior descending artery, were made, and the patient finally had an uneventful recovery. Careful study of the image in coronary angiography before the procedure and attentive examination of the plaque during the operation is the key to discriminating the variant vessel.
\end{abstract}

Keywords: accessory left anterior descending artery, coronary anomalies, cardiac surgery, off-pump coronary artery bypass

\section{Introduction}

Although the accessory left anterior descending artery (LAD) has been widely reported to be associated with congenital heart diseases such as tetralogy of Fallot and transposition of the great arteries, ${ }^{1,2)}$ it is rarely found to be the single coronary anomaly in adult with severe atherosclerosis.

\section{Case Report}

A 74-year-old man presenting with features of unstable angina was transferred to our hospital. His coronary risk factors included his age, addiction to smoking

Department of Cardiovascular Surgery, Shanghai Cardiovascular Institution and Zhongshan Hospital, Shanghai Fudan University, Shanghai, China

Received: February 28, 2012; Accepted: April 25, 2012 Corresponding author: Chunsheng Wang, MD. Department of Cardiovascular Surgery, Shanghai Cardiovascular Institution and Zhongshan Hospital, Shanghai Fudan University, No 180, Fenglin Road, Shanghai 200032, China

Email: zswangchunsheng@126.com

(C)2012 The Editorial Committee of Annals of Thoracic and Cardiovascular Surgery. All rights reserved. and alcohol for more than $30 \mathrm{yrs}$, and a history of hypertension. He complained of chest tightness and shortness of breath at midnight without alleviation until admitted to the local hospital in the morning. Coronary angiography revealed the whole course of the left coronary artery with severe calcification. There was 30\% stenosis at the middle and distal segment of the left main branch. The LAD had $30 \%-40 \%$ of narrowing at the proximal and middle portions, from which an accessory LAD, whose ostium was severely narrowed (99\%) as well as $80 \%$ stenosis at its middle segment, arose (Fig. 1A). The 1st diagonal branch (D1) was patent, but the left circumflex artery (LCX) had 70\% stenosis at the beginning and proximal segments and $85 \%$ narrowing at the origin of the 2nd obtuse marginal branch (OM2). The whole course of the right coronary artery also had pronounced calcification as well as total occlusion due to its middle segment.

An off-pump coronary artery bypass (OPCAB) was scheduled electively. During the procedure, we identified that the accessory LAD, a $2 \mathrm{~mm}$ vessel with a sense of plaque in touch at proximal and middle segment, ran in the anterior interventricular groove to the apex, which was the traditional course of the LAD, while the LAD only had a little portion, accompanied 


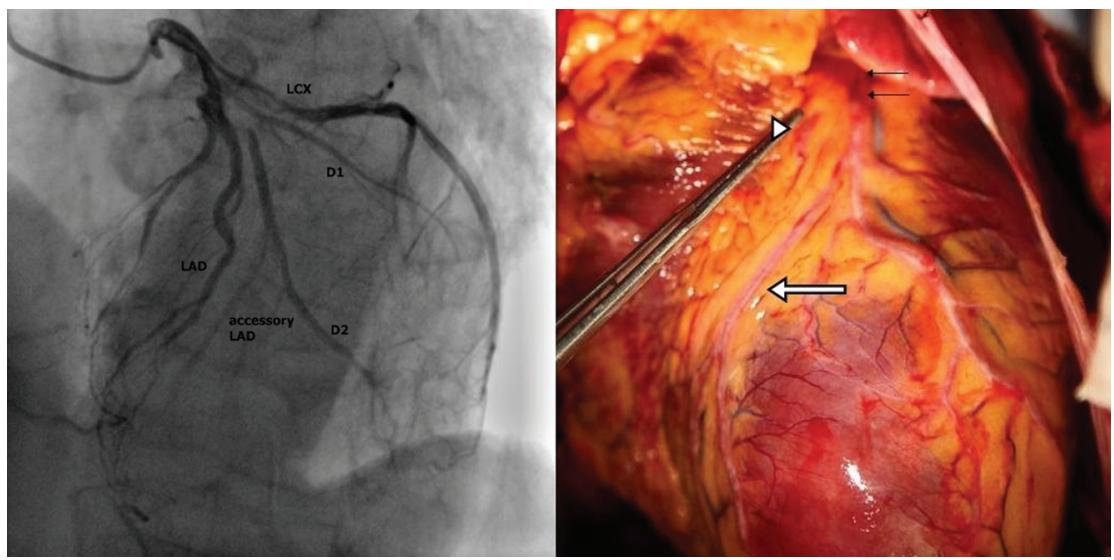

Fig. 1 Before the procedures (A) The coronary angiography demonstrated that the accessory LAD, whose ostium was critically narrowed (99\%), was given rise by the LAD. They paralleled with each other on their course for some distance. (B) The accessory LAD (arrow), a $2 \mathrm{~mm}$ vessel with a sense of plaque (double arrow) in touch at proximal and middle segment, ran in the anterior interventricular groove to the apex of the heart, which was the traditional course of the LAD, while the LAD (arrow-head) just had a little portion, accompanied by the accessory LAD, appearing on the surface of the heart. D1: 1st diagonal branch, D2: 2nd diagonal branch, LAD: left anterior descending artery, LCX: left circumflex artery

by the accessory LAD, appearing on the surface of the heart (Fig. 1B). The left internal mammary artery (LIMA), $1.5 \mathrm{~mm}$ in diameter, was harvested together with the saphenous vein graft (SVG) with a diameter of $4 \mathrm{~mm}$. We used a curved arm cardiac stabilization system with clear immobilizer (OPCAB Stabilization Elite System, Teleflex Medical, NC, USA) to locally stabilize the target vessels for each anastomosis. The LIMA was anastomosed to the distal segment of the accessory LAD with an 8-0 continuous polypropylene running suture, and intraoperative graft patency verification was performed by a transit time flow measurement (TTFM) (Medi-Stim Butterfly, Oslo, Norway). An adequate mean flow value $(40 \mathrm{ml} / \mathrm{min})$ and a pulsatility index (PI) of 3.1 were noted at a mean arterial pressure of $80 \mathrm{mmHg}$ (Fig. 2A). After that, the SVG was anastomosed sequentially to the 2nd diagonal branch (D2, originated from the accessory LAD), the OM2 and the posterior descending artery (PDA) with 7-0 continuous polypropylene running suture.

The patient's postoperative course was uneventful. No further complications occurred. After a postoperative coronary CT showing patency for the grafts (Fig. 2B), the patient was discharged on the 9th day after the surgery. A follow-up angiography performed at 7 months later also demonstrated good patency of
LIMA to accessory LAD bypass (Fig. 3), and the patient is still in good health.

\section{Discussion}

$\mathrm{OPCAB}$, which is believed to be an effective surgical technique in recent years, is being performed with increasing frequency. ${ }^{3)}$ It is reported that this emerging technique is associated with less systemic inflammatory response syndrome (SIRS), minor myocardial injury, attenuate renal damage and reduced neurological complications as compared with traditional on-pump surgery. ${ }^{4-7)}$ Thus, for those high-risk populations, such as an elderly heavy smoker with triple-vessel disease in the present case, we choose to perform OPCAB and assume that it may offer a superior outcome for the patient.

Apart from the advanced style of the procedure, early graft patency can influence either early or late outcome of the surgery as well. ${ }^{8)}$ TTFM is regarded as a promising method and has become popular for accessing graft patency intraoperatively. Although, till now, we still lack a universally acknowledged interpretation of the TTFM findings, a brief overview of the literatures suggested that the combination of the mean flow value and the PI may effectively judge 


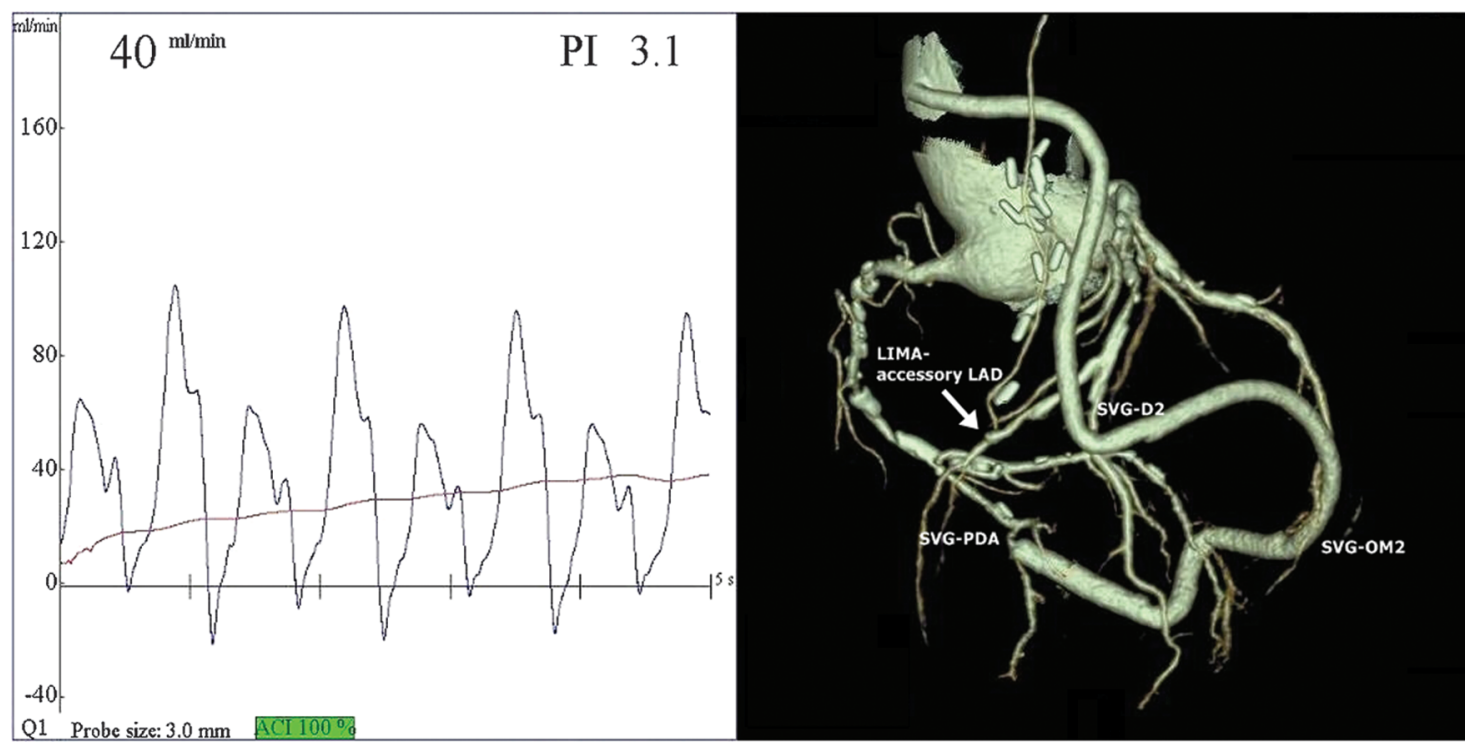

Fig. 2 After the procedures (A) TTFM findings in LIMA to accessory LAD graft suggested an adequate mean flow value and appropriate PI. (B) The postoperative CT showed grafts in a good run (LIMA-accessory LAD (arrow), Ao-SVG-D2-OM2-PDA). Ao: aorta; D2: 2nd diagonal branch; LAD: left anterior descending artery; LIMA: left internal mammary artery; OM2: 2nd obtuse marginal branch; PDA: posterior descending artery; PI: pulsatility index; SVG: saphenous vein graft; TTFM: transit time flow measurement

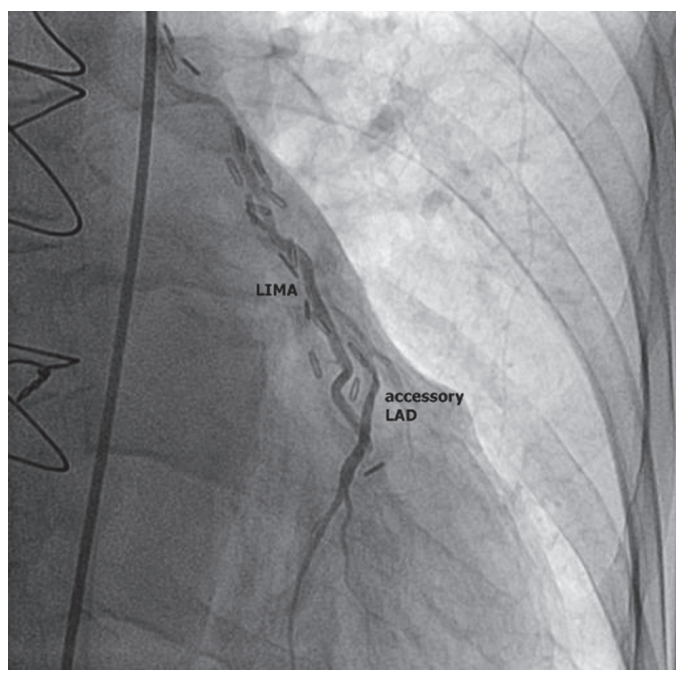

Fig. 3 A follow-up angiography performed at 7 months after the surgery also demonstrated good patency of LIMA to accessory LAD bypass. LAD: left anterior descending artery; LIMA: left internal mammary artery

graft failure. D'Ancona evaluated 409 patients with a total of 1145 grafts by TTFM. Twenty nine of the 37 abnormal grafts were revised for low mean flow values $(<15 \mathrm{ml} / \mathrm{min})$ and high PIs $(>5)$. After revision, mean flow values increased from a mean value of $6.58 \pm 6.00$ to $36.29 \pm 26.91 \mathrm{ml} / \mathrm{min}(P<0.0001)$, and the PIs also improved from $24.44 \pm 46.51$ to $2.80 \pm 1.68$ $(P<0.0001) .{ }^{9)}$ Similarly, Beran indicated that a mean flow value less than $20 \mathrm{ml} / \mathrm{min}$ along with a PI greater than 5 should induce the surgeon to re-evaluate the anastomosis and to redo it. ${ }^{10)}$ Recently, Tokuda defined the cut-off value for graft revision, in which the mean flow value and the PI were $15 \mathrm{ml} / \mathrm{min}$ and 5.1 for grafts to the left coronary artery. Both of them had a high negative predictive value $(>0.92)$, which means that the grafts with mean flow values greater than $15 \mathrm{ml} / \mathrm{min}$ and PIs less than 5.1 should be considered patent in all probability. ${ }^{11)}$

Careful study of the image in the coronary angiography before OPCAB is very important to distinguish the variant vessels. In the coronary angiogram, it is easy to seek out the LAD, which commonly develops from the left main artery and ends at the apex, on the edge of the cardiac contour in right anterior view. Usually, there are three septal and three diagonal branches originating at right and acute angles from it. ${ }^{12)}$ If you discover another vessel also deriving from the LAD and paralleling its normal course for some distance as well, this will mostly be the accessory LAD. ${ }^{13)}$ Sometimes, it may be a little bit difficult to discriminate this rare coronary anomaly from the D1 in the coronary angiogram. But their different journeys on the surface of the heart could 
be helpful to tell them apart. The pathway of the D1 is constantly to the anterolateral wall of the left ventricle, while the accessory LAD, as its name indicating, runs along with the LAD towards the apex. Besides, since its caliber and length is significantly larger and longer than those branches of the D1 or D2, it should not be a branch of them, either. Occasionally, surgeons may be confused by the similarity of the LAD and the accessory LAD during the procedure. We believe that the following experiences may be helpful to solve the vital problem. First, still in the coronary angiogram, surgeons carefully study the length, caliber, and configuration of both LAD and accessory LAD, try to find some relative marks, such as the pattern or the course of the diagonal branch, to help tell them apart, and analyze which one may run on the surface of the heart while the other in the interventricular groove. Secondly, in the procedures, surgeons examine the anatomy of the 2 vessels attentively and compare them with the images in coronary angiography. Thirdly, for the patients with apparent atherosclerosis, touching the plaque as well as looking into its shape and position may provide wonderful assistance to uncover the diseased artery. Last but not least, after we incising the vessel, an intracoronary bougie may be helpful to detect the location and the degree of the stenosis so as to check our estimation before. Besides, impairment of the intimae should be avoided.

Isolated CA anomalies are rare in the adult population. ${ }^{14)}$ However, under the circumstance of the needs for regeneration, such as in the setting of coronary artery disease, both vasculogenesis and angiogenesis are now known to actively occur within the adult heart, ${ }^{15)}$ which may contribute to the variation of the CA in adult. We assume that, as long as atherosclerosis emerged at the LAD, the collateral circulation would be produced and expanded to re-connect the proximal and distal segments over the stenosis. ${ }^{12)}$ Thus, the new connection might turn out to be the fresh LAD while the former one should be the kinking accessory LAD we recognize today.

\section{Conclusion}

We report a rare case of accessory LAD in adult with severe atherosclerosis, and careful study of the image in coronary angiography before the procedure and attentive examination of the plaque during the operation is the key to discriminating the variant vessel.

\section{Disclosure Statement}

We have no financial or other interest in the manufacture or distribution of the device concerned in the case report.

\section{References}

1) Shrivastava S, Mohan JC, Mukhopadhyay S, et al. Coronary artery anomalies in tetralogy of Fallot. Cardiovasc Intervent Radiol 1987; 10: 215-8.

2) Taylor AM, Dymarkowski S, Hamaekers P, et al. MR coronary angiography and late-enhancement myocardial MR in children who underwent arterial switch surgery for transposition of great arteries. Radiology 2005; 234: 542-7.

3) Natarajan A, Samadian S, Clark S. Coronary artery bypass surgery in elderly people. Postgrad Med J 2007; 83: $154-8$.

4) Murphy GJ, Ascione R, Angelini GD. Coronary artery bypass grafting on the beating heart: surgical revascularization for the next decade? Eur Heart J 2004; 25: 2077-85.

5) van Dijk D, Nierich AP, Jansen EW, et al. Early outcome after off-pump versus on-pump coronary bypass surgery: results from a randomized study. Circulation 2001; 104: 1761-6.

6) Loef BG, Epema AH, Navis G, et al. Off-pump coronary revascularization attenuates transient renal damage compared with on-pump coronary revascularization. Chest 2002; 121: 1190-4.

7) Ricci M, Karamanoukian HL, Abraham R, et al. Stroke in octogenarians undergoing coronary artery surgery with and without cardiopulmonary bypass. Ann Thorac Surg 2000; 69: 1471-5.

8) Yusuf S, Zucker D, Peduzzi P, et al. Effect of coronary artery bypass graft surgery on survival: overview of 10-year results from randomised trials by the Coronary Artery Bypass Graft Surgery Trialists Collaboration. Lancet 1994; 344: 563-70.

9) D'Ancona G, Karamanoukian HL, Ricci M, et al. Graft revision after transit time flow measurement in offpump coronary artery bypass grafting. Eur J Cardiothorac Surg 2000; 17: 287-93.

10) Beran E, Kapitan M, Mächler H, et al. Accurate preoperative echocardiography has more impact on prediction of long-term mortality than intra-operatively measured flow in coronary bypass grafts. Eur J Cardiothorac Surg 2011; 40: 245-8.

11) Tokuda $Y$, Song MH, Ueda $Y$, et al. Predicting early coronary artery bypass graft failure by intraoperative transit time flow measurement. Ann Thorac Surg 2007; 84: 1928-33.

12) Fioranelli M, Gonnella C, Tonioni S. Clinical Anatomy of the Coronary Circulation. In: Pavone P, Fioranelli 
M, Dowe DA, Gonnella C, Tonioni S. CT Evaluation of Coronary Artery Disease. Milan: Springer, 2009; pp 1-13.

13) Siwik ES, Erenberg F, Zahka KG. Tetralogy of Fallot. In: Allen HD, Driscoll DJ, Shaddy RE, Feltes TF eds.; Moss and Adams' heart disease in infants, children, and adolescents: including the fetus and young adult.
Philadelphia, PA: Lippincott Williams\&Wilkins, 2008; p 893.

14) Asha M, Sriram R, Mukundan S, et al. Single coronary artery from the left sinus with atherosclerosis. Asian Cardiovasc Thorac Ann 2003; 11: 163-4.

15) Smart N, Dubé KN, Riley PR. Coronary vessel development and insight towards neovascular therapy. Int $\mathrm{J}$ Exp Pathol 2009; 90: 262-83. 